\title{
UM CONTROLADOR DE CORRENTE DE CARGA PARA O CONVERSOR EM MATRIZ TRIFÁSICO PARA TRIFÁSICO E PARA O INVERSOR DE FREQUÊNCIA SEM CAPACITOR DO ELO DE CORRENTE CONTÍNUA
}

\author{
M. E. Oliveira Filho* \\ mfilho@dsce.fee.unicamp.br \\ J. R. Gazoli* \\ gazoli@gmail.br
}

\author{
A. J. Sguarezi Filho* \\ sguarezi@dsce.fee.unicamp.br \\ E. Ruppert * \\ ruppert@fee.unicamp.br
}

*Universidade Estadual de Campinas, FEEC-DSCE

Av. Albert Einstein, 400

Cidade Universitária Zeferino Vaz, Campinas, SP

\begin{abstract}
A Controller for the Three-Phase to Three-Phase Matrix Converter and for the Voltage Source ThreePhase Inverter Without DC-Link Capacitor

Voltage source three-phase inverter plays an important role in modern industry. The conventional voltage source inverter has a large electrolytic capacitor as energy store element in order to keep the DC-link voltage constant. However, a large electrolytic capacitor increases the input current distortion. Replacing the large electrolytic capacitor by a small film capacitor, the input current quality is improved but the DC-link voltage and the output currents are distorted by low order harmonic components.
\end{abstract}

The three phase to three phase matrix converter performs electric energy direct transfer from the input to the output with sinusoidal input current although the distortion and the unbalance of the input voltages affects directly the output of the matrix converter.

\footnotetext{
Artigo submetido em 25/08/2009 (Id.: 01028)

Revisado em 10/11/2009, 30/12/2009, 04/03/2010

Aceito sob recomendação do Editor Associado Prof. Darizon Alves de An-
} drade
This paper proposes a load current control method to the matrix converter and also to the voltage source inverter in absence of the dc-link capacitor in order to compensate the distortion caused by low order harmonics components in the output currents. The proposed load current control scheme employs just one PI controller and since that the space vector modulation is used in this control, the switching frequency of the converter is kept constant. Theorical foundation, simulations results and experimental results are presented.

KEYWORDS: matrix converter, voltage source inverter, modulation index,current control, bidirectional switch, space vector, input filter.

\section{RESUMO}

Com o avanço da tecnologia dos dispositivos semicondutores de potência os inversores de frequência atuam de forma importante em toda indústria. O inversor de frequência convencional contém elementos armazenadores de energia no seu elo de corrente contínua. Estes elementos armazenadores de energia são capacitores eletrolíticos e possibilitam manter a tensão do elo de corrente contínua constante. Entretanto, quanto maior sua capacitância maior é a distorção da corrente de entrada. 
Com a substituição do banco de capacitores eletrolíticos por um capacitor de filme, diminui-se a distorção nas correntes de entrada, porém distorções indesejadas aparecem na tensão do elo de corrente contínua e também nas correntes de saída. Estas distorções são compostas por componentes harmônicas de baixas frequências.

O conversor em matriz trifásico-trifásico por sua vez, possibilita a transferência direta de energia da entrada (rede) diretamente para a saída (carga) sem a necessidade de elementos armazenadores de energia como acontece no inversor de frequência. Entretando o desbalanceamento das tensões da entrada afetam diretamente a forma de onda das tensões da saída.

Dessa maneira, este trabalho propõe um método de controle de corrente do conversor em matriz e do inversor de frequência sem banco de capacitores do elo de corrente contínua com objetivo de compensar o desbalanceamento das tensões de saída do conversor em matriz e compensar também as componentes harmônicas de baixa frequência que aparecem nas correntes de saída do inversor de frequência. O método proposto utiliza um controlador do tipo PI e modulação por vetores espaciais para que a frequência de acionamento das chaves dos conversores seja constante.

PALAVRAS-CHAVE: conversor em matriz, inversor de frequência, índice de modulação, controle de corrente, chave bidirecional, filtro de entrada.

\section{INTRODUÇÃO}

Com o avanço da tecnologia dos dispositivos semicondutores os conversores são amplamente empregados em aplicações industriais eletrônicos de potência. O conversor mais empregado é o inversor de frequência tipo fonte de tensão que é composto por um retificador trifásico não controlado e um inversor trifásico controlado por IGBTs ou MOSFETs ambos compartilhando o mesmo elo de corrente contínua onde é instalado um banco de capacitores eletrolíticos que eliminam as oscilações de baixa frequência causadas pelo processo de retificação (Rashid, 2004).

Um conversor eletrônico de potência que tem recebido atenção devido ao fato de ser uma alternativa ao inversor de frequência fonte de tensão com banco de capacitores no elo de corrente contínua é o conversor indireto ca/cc/ca (Malesani et al., 1995) que consiste de um retificador-inversor PWM com um banco de capacitores do elo de corrente contínua com reduzida capacitância. Neste caso, o capacitor eletrolítico é substituído por um capacitor não eletrolítico com capacitância muito menor e com esta substituição, a distorção harmônica total da corrente de entrada é reduzida drásticamente e a confiança no conversor é aumentada. O conversor indireto é apresentado na Fig. 1. Por outro lado, com a retirada do elemento armazenador de energia aparecerão flutuações indesejadas na tensão do elo de corrente contínua do conversor com frequência igual a seis vezes a frequência da rede elétrica. Além disso, a ondulação das flutuações aumentarão caso a fonte apresente componentes harmônicos de baixa ordem ou esteja desbalanceada. Dessa forma, o controle do conversor indireto ca/cc/ca é complexo e indutores de alta indutância são necessários para reduzir as ondulações de corrente (Choi e Sul, 1997).

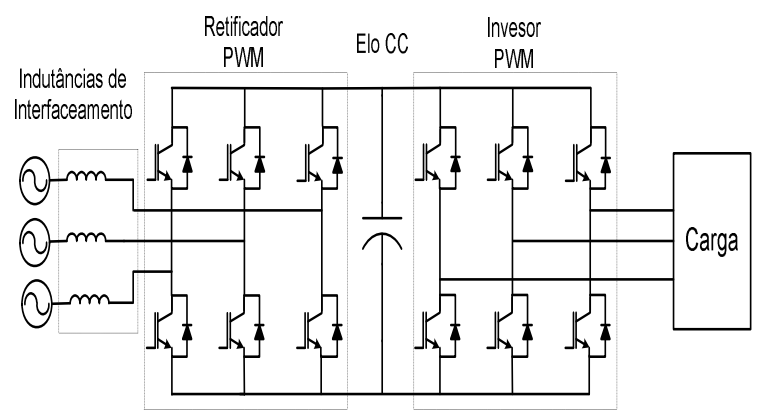

Figura 1: Conversor indireto $\mathrm{ca} / \mathrm{cc} / \mathrm{ca}$.

Um outro conversor que tem se destacado é o conversor em matriz pois não utiliza elo de corrente e dispositivos armazenadores de energia. O conversor em matriz pode transferir energia diretamente da rede para a carga sem a necessidade de intermediação de um elo de corrente contínua e, dessa forma, torna-se possível sua operação nos quatro quadrantes, fator de potência unitário, tensões e correntes com formas senoidais e componentes harmônicos de alta frequência tanto no lado da carga como no lado da fonte. Estes recursos fazem do conversor em matriz uma alternativa interessante ao tradicional inversor de frequência fonte de tensão (Nielsen, Blaabjerg e Pedersen, 1996; Kwak et al., 2002; Zhang et al., 2001).

Um modelo simplificado da topologia do conversor em matriz (CM) trifásico é apresentado na Fig. 2, sendo que as chaves $S_{11}$ a $S_{33}$ são de potências bidirecionais , $v_{i l}(t)$, $v_{i 2}(t)$ e $v_{i 3}(t)$ são as fontes de tensões de entrada e $v_{o 1}(t)$, $v_{o 2}(t)$ e $v_{3 o}(t)$ são as tensões de saídas desejadas. Como observado nas Figs. 1 e 2 o conversor indireto ca/cc/ca e o conversor em matriz demandam mais chaves de potência e esforço computacional para implementação de estratégias de controle. Como resultado, os conversores ainda não são rentáveis porém resultados interessantes de implementação da modulação por vetores espaciais num retificador-inversor (Chen e Kazerani, 2006) e num conjunto de dois inversores em cascata são apresentados 
em (Chen e Kazerani, 2006) e (Liu et al., 2007) respectivamente. Um método alternativo de implementação do conversor em matriz é o conversor em matriz indireto que consiste em pontes separadas para a rede e para a carga (Jussila e Tuusa, 2007).

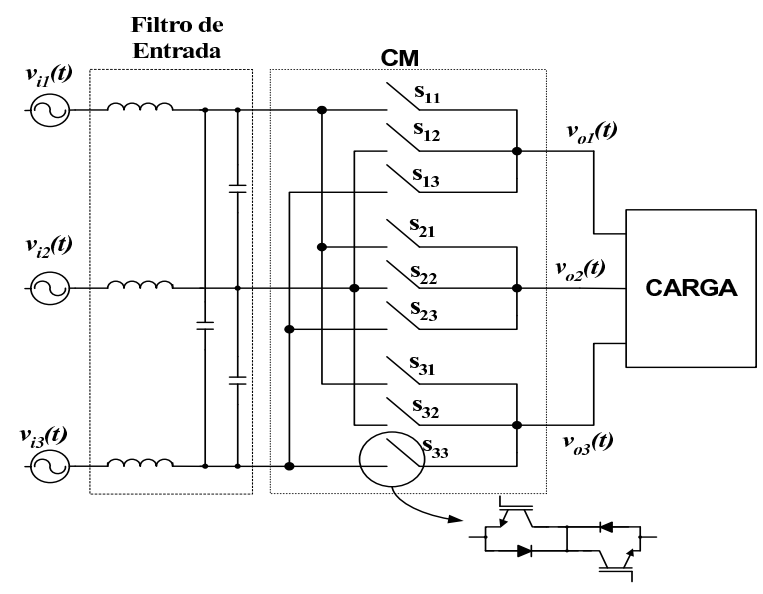

Figura 2: Conversor em matriz trifásico.

A ausência de elevados elementos reativos no CM é uma vantagem sobre o conversor de frequência tradicional. Contudo, esta ausência possibilita que a distorção das tensões de entrada sejam imediatamente refletidas para o lado da carga (Zanchetta et al., 2008).

Resultados que mostram a redução da influência das tensões de entrada distorcidas e desbalanceadas do CM são apresentados em (Casadei et al., 1998; Nielsen, Casadei, Serra e Tani, 1996; Kang et al., 2002; Vincenti e Ziogas, 1992) e a na maior parte deles é realizada a medição da tensão de entrada a cada período de amostragem para o cálculo do índice de modulação. Normalmente a decomposição da tensão de entrada em componentes simétricos e a transformação para o referencial síncrono são necessárias para a implementação destas estratégias. Alguns trabalhos empregam uma malha de controle de corrente para melhorar o desempenho do CM (Mutschler e Marcks, 2002; Wiechmann et al., 1997; Sato et al., 2007). Outra possibilidade é a otimização do uso dos vetores nulos (Casadei et al., 2009).

Com relação ao controle de corrente do inversor de frequência que atua de forma importante em aplicações de alta potência como nos acionamentos eletrônicos de motores, e em filtros ativos de potência. Muitos tipos de controladores de corrente podem ser encontrados na literatura. Dois tipos de controladores são extensivamente empregados devido às suas simplicidades. Os controladores são: controladores por histerese e controlador por comparação em rampa (Malesni e Tomasin, 1993; Ta- keshita e Shimada, 2008).

O controlador por histerese apresenta boa precisão e robustez pois mantém o erro, entre a referência e o valor medido, dentro de uma banda especificada. Sua maior desvantagem é devido a frequência variável do acionamento das chaves do conversor. No controlador de comparação por rampa, o erro da corrente instantânea é injetado num controlador do tipo PI que gera a tensão de comando que é comparada a uma onda triangular na frequência de chaveamento desejada, porém o valor da amplitude da tensão não deve exceder a inclinação do triangulo da onda e o controlador PI não produz erro nulo de corrente. O desempenho do controlador de comparação com rampa pode ser aumentado se a transformação para o referencial síncrono (dq) for adotado.

Neste trabalho propõe-se um método de controle de corrente para o CM e para o inversor de frequência sem o banco de capacitores do elo de corrente contínua com objetivo de encontrar correntes de saídas sem distorções. O controlador possibilita corrente senoidal na saída $\mathrm{CM}$ ou do inversor de frequência com a ausência do banco de capacitores do elo de corrente contínua mesmo que as tensões de entrada do conversor estejam desbalanceadas ou distorcidas. O controle de corrente de carga é implementado com um controlador PI e outra vantagem de utilizar o controle de corrente de carga é sua menor complexidade na proteção de sobrecorrentes no CM desde que a corrente da saída seja medida diretamente. Resultados de simulação e experimentais são apresentados para a validação do método de controle proposto.

\section{O MÉTODO DE CONTROLE DE COR- RENTE DE CARGA PROPOSTO}

O método de controle de corrente utiliza a módulo da corrente da carga que é encontrada a partir do vetor espacial da corrente de saída $I_{o}$ que é dado por (1).

$$
\left|I_{o}\right|=\sqrt{i_{o a}^{2}(t)+i_{o b}^{2}(t)+i_{o c}^{2}(t)}
$$

Sendo $i_{o a}(t), i_{o b}(t)$ e $i_{o c}(t)$ as correntes de fase $a b c$. Quando a tensão de entrada for desbalanceada ou distorcida, indesejados componentes harmônicos de baixa ordem aparecerão na corrente de saída. Neste caso particular, as correntes de saída são modeladas por (2). 


$$
\begin{array}{r}
i_{o 1}=I_{p} \sin (\omega t+\phi)+I_{n} \sin (\omega t+\phi)+ \\
+I_{h} \sin \left(\omega_{h} t+\phi\right) \\
i_{o 2}=I_{p} \sin \left(\omega t+\phi-\frac{2 \pi}{3}\right)+ \\
+I_{n} \sin \left(\omega t+\phi+\frac{2 \pi}{3}\right)+I_{h} \sin \left(\omega_{h} t+\phi-\frac{2 \pi}{3}\right) \\
i_{o 3}=I_{p} \sin \left(\omega t+\phi+\frac{2 \pi}{3}\right)+ \\
+I_{n} \sin \left(\omega t+\phi-\frac{2 \pi}{3}\right)+I_{h} \sin \left(\omega_{h} t+\phi+\frac{2 \pi}{3}\right)
\end{array}
$$

Em (2), $p$ corresponde aos componentes de sequência positiva, $n$ correspondem aos componentes de sequência negativa e $h$ são os componentes harmônicos. O módulo do vetor espacial é calculada com a utilização de (1) e (2) e os resultados são apresentados em (3) depois de algumas manipulações algébricas realizadas.

$$
\begin{array}{r}
\left|I_{o}\right|=\left(I_{p}^{2}+I_{n}^{2}+I_{h}^{2}-2 I_{p} I_{n} \cos (2 \omega t)+\right. \\
\left.+2 I_{p} I_{h} \cos [(h-1) \omega t]-2 I_{n} I_{h} \cos [(h+1) \omega t]\right)^{0.5}
\end{array}
$$

A equação (3) mostra que o módulo do vetor espacial $I_{o}$ oscilará devido aos componentes de sequência negativa e harmônicas da corrente de saída. Então, no método proposto as correntes de saída do conversor medidas são usadas para calcular o módulo do vetor espacial $I_{o}$ através de (1) e este resultado é comparado com a corrente de referência $I_{r e f}$. O erro de corrente instantâneo é injetado em um controlador PI que gera o índice de modulação $m$ para a modulação por vetores espaciais, permitindo que as tensões de saídas sejam geradas. Se as tensões de entrada forem senoidais e balanceadas, a corrente de saída do conversor será senoidal. Nesta condição o módulo dos vetores espaciais $V_{i}$ and $I_{o}$ são constantes. Dessa maneira, pode-se visualizar o CM como um conversor $\mathrm{CC}$ e um controlador do tipo PI pode ser usado no método de controle de corrente de carga proposto para garantir que o erro de regime seja nulo. O método de controle de corrente proposto é apresentado na Figura 3 para o $\mathrm{CM}$ e na Fig 4 para o inversor de frequência com a ausência do banco de capacitores do elo de corrente contínua.

A expressão do controlador que possibilita o cálculo do índice de modulação a partir do erro de corrente instantânea e do controlador PI é mostrada na equação (4).

$$
m=\operatorname{sat}_{0}^{0.866}\left[k_{p}\left(I_{\text {ref }}-\left|I_{o}\right|\right)+k_{i} \int\left(I_{\text {ref }}-\left|I_{o}\right|\right) d t\right]
$$

sendo que $k_{p}$ é o ganho proporcional, $k_{i}$ é o ganho integral e $I_{r e f}$ é a corrente de referência.

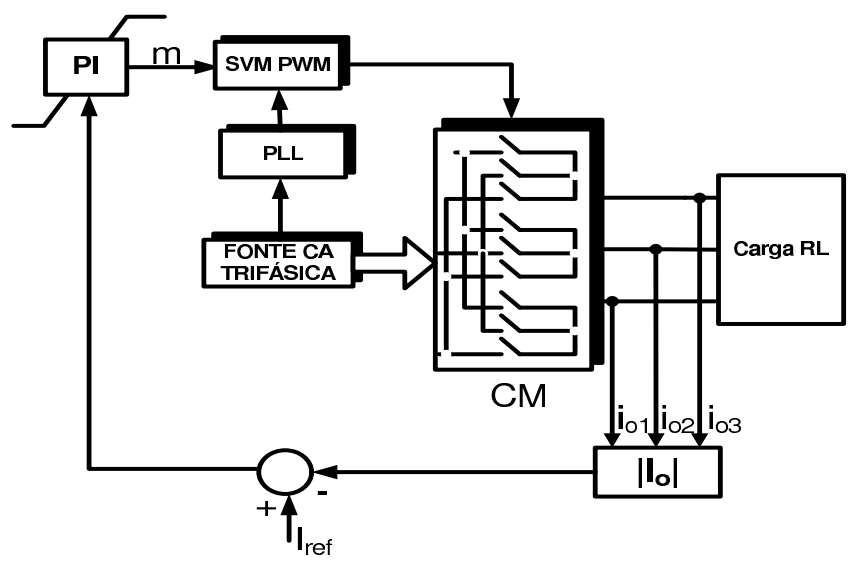

Figura 3: O método de controle de corrente de carga proposto para o $\mathrm{CM}$.

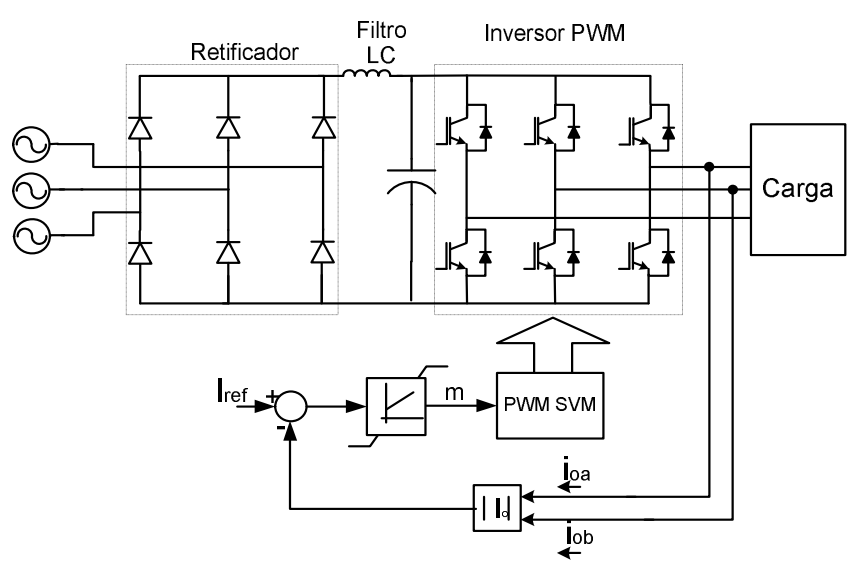

Figura 4: O método de controle de corrente de carga proposto para o inversor de frequência sem banco de capacitores no elo de corrente contínua.

O projeto do parâmetros do controlador PI são encontrados no algoritmo apresentado em (Basilio e Matos, 2002).

Desta forma se a malha do controlador de corrente de carga forçar o módulo do vetor espacial $I_{o}$ seguir a referência, a corrente de saída não terá componentes harmônicos de baixas frequências e também não terá distorções. Isto é realizado pelo controlador PI que corrige dinamicamente o índice de modulação a fim de que o módulo do vetor espacial $I_{o}$, que é calculado a partir de (1), atenda ao valor da corrente de referência $I_{r e f}$. Assim, o controlador de corrente proposto possibilita que a corrente da carga seja senoidal sem a necessidade de transformação de coordenadas o que reduz o custo computacional. 


\subsection{Modulação em largura de pulso para o conversor em matriz}

Com a utilização da modulação em largura de pulso por vetores espaciais no controle de corrente de carga obtémse frequência de chaveamento constante. Este tipo de modulação é empregado no controle do CM devido a sua simplicidade em realizar o cálculo do ciclo de comutação e prover tensões de saída balanceadas com o valor máximo de ganho do CM que é de 0,866 .

Com objetivo de empregar esta técnica de modulação o $\mathrm{CM}$ é modelado como dois conversores conectados através de um elo de corrente contínua imaginário: o primeiro estágio se comporta como um retificador de entrada com elo de corrente contínua e o segundo como inversor de frequência tipo fonte de tensão. Esta aplicação possibilita usar a conhecida técnica de modulação por vetores espaciais em ambos os estágios como é apresentado em (Hubber e Borojevic, 1995). Uma outra possibilidade interessante de modulação foi apresentada em (Odaka et al., 2009) e a estratégia de modulação em largura de pulso escalar foi apresentada por (Accioly et al., 2007).

Fisicamente, as mesmas chaves bidirecionais do CM realizam a retificação e a inversão simultaneamente o que resulta em 36 possíveis combinações do vetor espacial da tensão de saída $V_{o}$ e do vetor espacial da corrente de entrada $I_{i}$. Então a partir dos vetores $I_{i}$ e $V_{o}$ obtém-se o vetor tensão de saída desejado $V$, os tempos de chaveamento são dados por (5) como mostrado em (Hubber e Borojevic, 1995), sendo que $T_{s}$ é o período de amostragem e $m$ é o índice de modulação.

$$
\begin{array}{r}
t_{a c}=T_{s} m \sin \left(60^{\circ}-\theta_{c}\right) \sin \left(60^{\circ}-\theta_{v}\right) \\
t_{a d}=T_{s} m \sin \left(60^{\circ}-\theta_{c}\right) \sin \left(\theta_{v}\right) \\
t_{b d}=T_{s} m \sin \left(\theta_{c}\right) \sin \left(\theta_{v}\right) \\
t_{b c}=T_{s} m \sin \left(\theta_{c}\right) \cdot \sin \left(60^{\circ}-\theta_{v}\right) \\
t_{o}=T_{s}-t_{a c}-t_{a d}-t_{b d}-t_{b c}
\end{array}
$$

O ângulo da corrente de entrada $\theta_{c}$ pode ser obtido através de um PLL (Blaabjerg et al., 2006) ou através da detecção de cruzamento por zero da tensão de fase de entrada. Desta forma, o vetor corrente de entrada será sincronizado com a tensão de fase da entrada. O ângulo da tensão de saída $\theta_{v}$ é calculado de acordo com a frequência de saída desejada da tensão de saída.

Como a modulação por vetores espaciais é flexível na escolha da sequência dos vetores de chaveamento para o CM, neste trabalho adotou-se a sequência apresentada na Figura 5. Os tempos utilizados nesta sequência são calculados de acordo com a Equação (5).

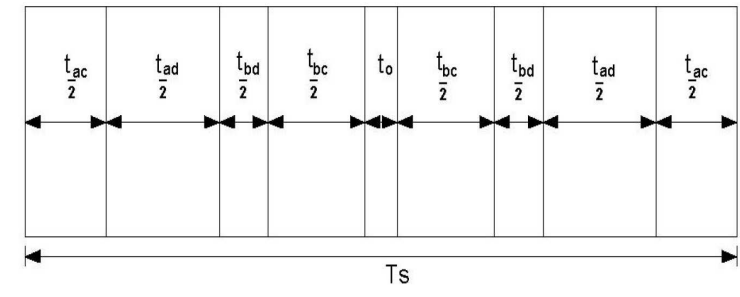

Figura 5: Sequência de chaveamento para modulação por vetores espaciais.

\subsection{Modulação por largura de pulso para o inversor de frequência fonte de tensão}

A modulação por vetores espaciais (MPE) tem sido escolhida para o controle de inversores fonte de tensão desde a metade da década de 80 (Holtz, 1992). A vantagem do uso do MPE é devido à sua simplicidade para realizar o cálculo dos tempos de comutação das chaves. Os ciclos $d_{a}, d_{b}$ e $d_{0}$ podem ser obtidos a partir da equação (6).

$$
\begin{array}{r}
d_{a}=m \sin \left(60^{\circ}-\theta\right) \\
d_{b}=m \sin (\theta) \\
d_{o}=1-d_{a}-d_{b}
\end{array}
$$

sendo $m$ o índice de modulação e $\theta$ é o ângulo do vetor espacial da tensão de saída desejada dentro do setor considerado.

A fim de evitar a influência das variações do elo de corrente contínua sobre a carga, os ciclos de comutação necessitam ser ajustados. A partir de (6), existem dois parâmetros de controle que possibilitam modificar os ciclos de comutação: o ângulo $\theta$ e o índice de modulação $m$. O ângulo $\theta$ controla a frequência da tensão de saída e este ângulo pode ser encontrado a partir da integração da frequência de saída desejada (Blaabjerg et al., 2006). Já o índice de modulação está relacionado com a módulo da tensão de saída e permite realizar a compensação das variações de tensão que podem ocorrer no elo de corrente contínua. Esta aplicação foi apresentada em (Enjeti e Shireen, 1992; Chen e Kazerani, 2006) e também foi aplicada ao CM em (Nielsen, Blaabjerg e Pedersen, 1996). Outra possibilidade de modulação é apresentada em (Chen e Kazerani, 2006) e nela a medição da tensão do elo de corrente contínua em cada período de chaveamento é realizada para o cálculo do índice de modulação através da relação entre a amplitude da tensão desejada e da tensão do elo de corrente contínua o que possibilita que esta modulação seja empregada em acionamentos nos quais o controle de tensão é realizado. 


\section{RESULTADOS DE SIMULAÇÃO}

\subsection{Conversor em Matriz}

A fim de verificar o desempenho do controlador de corrente proposto apresentado na Figura 3, um modelo do CM foi implementado no aplicativo computacional Matlab/SimPowerSystems com o emprego de chaves ideais. Uma carga trifásica indutiva conetada em estrela com $\mathrm{R}=5 \Omega$ and $\mathrm{L}=2 \mathrm{mH}$ por fase foi utilizada.

A frequência de chaveamento do CM usada foi de 10 $\mathrm{kHz}$ e a tensão de linha de entrada de $220 \mathrm{~V}, 60 \mathrm{~Hz}$. A tensão de entrada foi distorcida depois do instante de tempo $\mathrm{t}=30 \mathrm{~ms}$ com $10 \%$ da sequência negativa e $10 \%$ da componente de $5^{\circ}$ harmônica como mostrado na Figura 6. Na ausência do método de compensação, as correntes de saída apresentaram distorções quando a tensão de entrada se tornou distorcida depois de $t=30$ ms como mostrado em 7 .

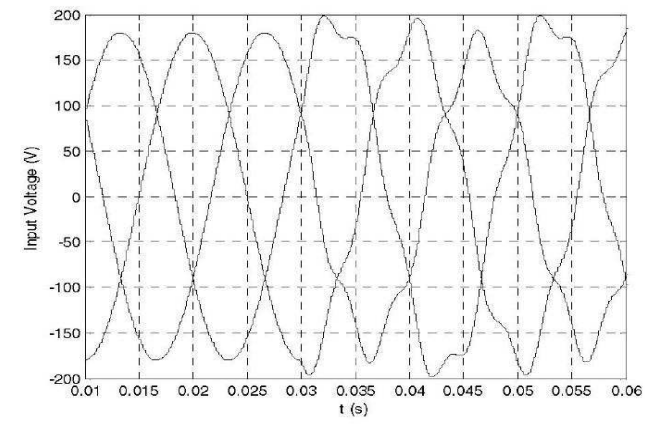

Figura 6: Tensão de fase de entrada do CM.

O controlador de corrente proposto foi aplicado ao CM e o índice de modulação foi corrigido dinamicamente pelo controlador PI afim de garantir que as correntes da carga se tornassem senoidais e balanceadas. O índice de modulação é apresentado na Figura 8 e as correntes da carga são apresentadas na Figura 9.

A Figura 10 mostra uma entrada em degrau que se altera de $10 \mathrm{~A}$ para $20 \mathrm{~A}$ no instante de tempo $\mathrm{t}=60 \mathrm{~ms}$ e a resposta dinâmica satisfatória do controlador pode ser observada devido ao fato de que as correntes e tensões da carga não apresentam distorções. A tensão de saída e sua componente fundamental são apresentadas na Figura 11 e a corrente de saída e seu espectro harmônico são apresentados na Figura 12.

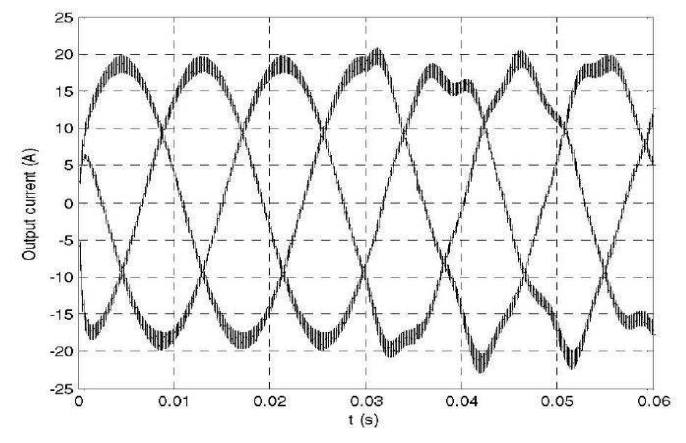

Figura 7: Correntes de carga do CM sem o controlador de corrente.

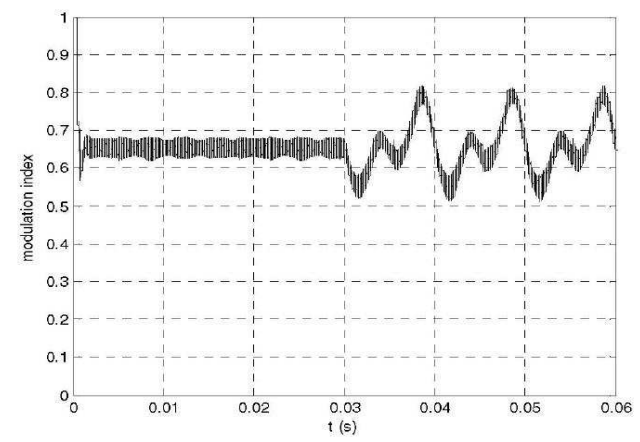

Figura 8: Índice de modulação gerado pelo controlador PI.

\subsection{Inversor de frequência tipo fonte de tensão sem banco de capacitores do elo de corrente contínua}

A simulação do controlador proposto apresentada na Figura 4 foi implementada com o emprego do aplicativo Matlab/Simulink/Simpowersystems e o algoritmo de controle é visto em (4). Os componentes do filtro são o mesmos utilizados no protótipo apresentado na próxima seção. A carga RL trifásica conectada em estrela apresenta como parâmetros: $\mathrm{R}=10 \Omega$ e $\mathrm{L}=6 \mathrm{mH}$. A frequência de chaveamento do inversor é $10 \mathrm{kHz}$ e a valor da tensão de linha da entrada é $220 \mathrm{~V}, 60 \mathrm{~Hz}$. Da Figura 13 até a Figura 15 são apresentados o desempenho satisfatório do inversor sem banco de capacitores de elo de corrente contínua com o controlador proposto quando as tensões de entrada não apresentam distorções e a referência de corrente é de $10 \mathrm{~A}$.

A resposta transitória do controlador foi obtida por simulação e está apresentada na Figura 16. Neste caso, a entrada em degrau de corrente de referênca é alterada de $5 \mathrm{~A}$ para $10 \mathrm{~A}$ e a tensão de entrada é distorcida no instante $\mathrm{t}=20 \mathrm{~ms}$ com $10 \%$ do componente de sequência 


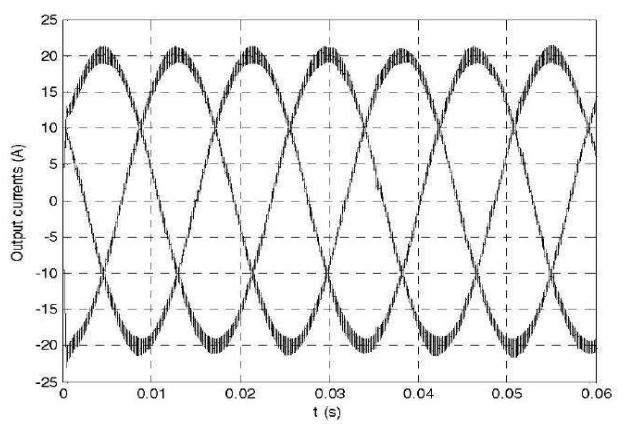

Figura 9: Correntes da carga com controle de corrente aplicado ao $\mathrm{CM}$.
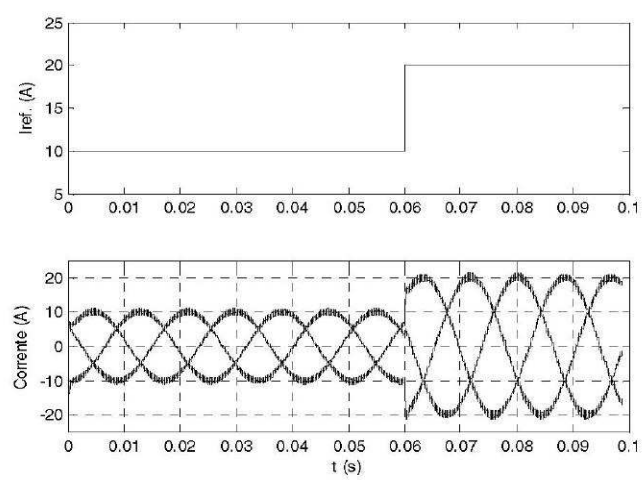

Figura 10: Resposta transitória do MC com o controlador de corrente proposto.

negativa e $10 \%$ do componente de $5^{\circ}$ harmônica. O desempenho satisfatório do controlador pode ser observado devido ao fato da referência de corrente ser atendida e a corrente da carga não apresentar distorções.

\section{IMPLEMENTAÇÃO DO PROTÓTIPO}

O controlador de corrente proposto foi implementado num inversor de frequência fonte de tensão e dois protótipos de baixa potência foram construídos empregando os mesmos circuitos de controle para os acionamentos das chaves como mostrado na Figura 17. Um dos protótipos é um inversor tradicional com banco de capacitores composto por 6 capacitores eletrolíticos que resulta numa capacitância de $1500 \mu \mathrm{F}$. O outro protótipo é um inversor de frequência que teve seu banco de capacitores do elo de corrente contínua trocados por um filtro LC que possibilita remover as componentes harmônicas de alta frequência geradas pelo chaveamento. Tanto a indutância quanto a capacitância tem valores pequenos. A construção destes protótipos foi realizada para a comparação dos custos de construção e desempenho.

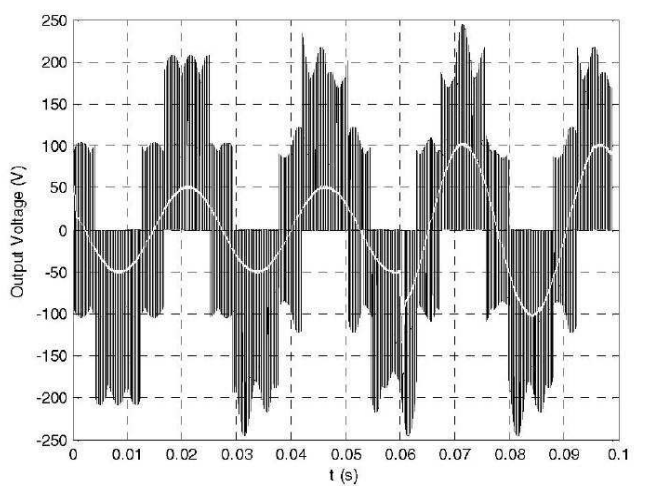

Figura 11: Tensão de linha de saída gerada pelo CM.

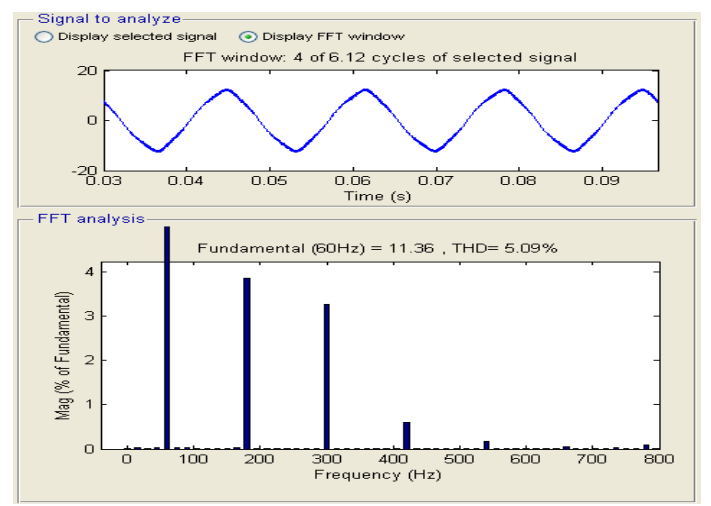

Figura 12: Corrente de entrada do CM e seu espectro harmônico.

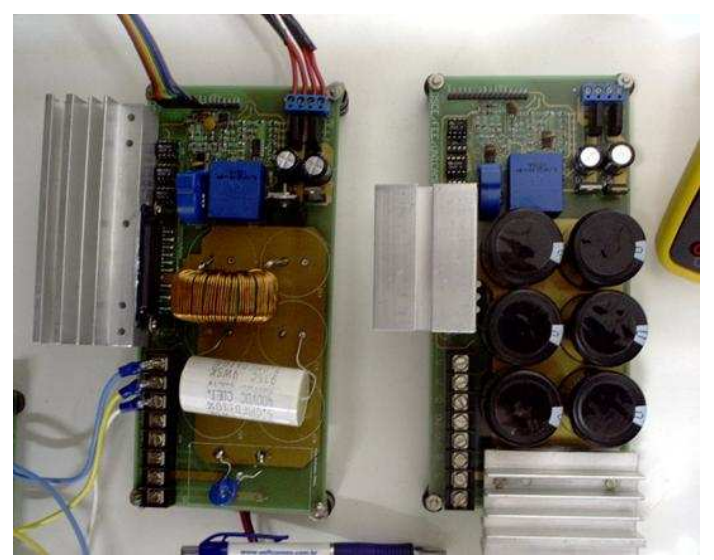

Figura 17: Protótipos dos inversores: com filtro LC (esq.) e com banco de capacitores (dir.)

\subsection{Projeto do filtro LC}

O filtro LC pode ser modelado com a utilização de um circuito harmônico equivalente como apresentado na Fi- 


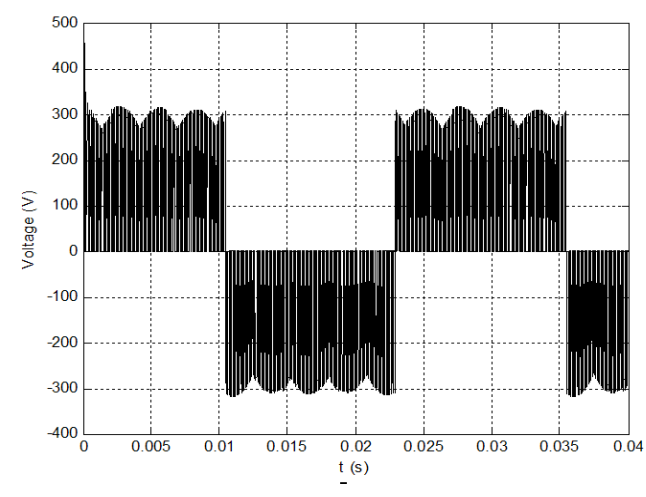

Figura 13: Tensão de fase de saída do inversor.

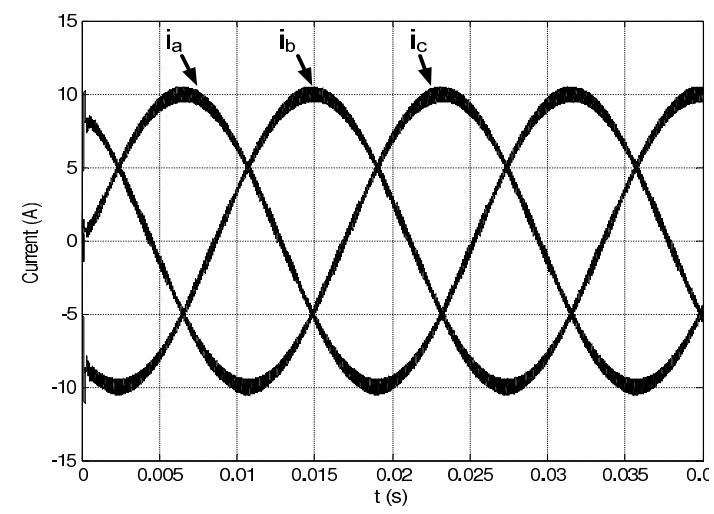

Figura 14: Correntes da carga do inversor sem capacitores no elo de corrente contínua com o controlador de corrente proposto.

gura 18. O armortecimento $\xi$ e a frequência natural $\omega_{n}$ são dados por (8).

$$
\xi=\frac{R_{d}}{2 \omega_{n} L}=\frac{R_{d}}{2} \sqrt{\frac{C}{L}}
$$

sendo que $\omega_{n}=\frac{1}{\sqrt{L C}}$ e $R_{d}$ é o valor do resistor de amortecimento.

No protótipo construído, os componentes do filtro LC são um indutor toroidal com núcleo de ferro de $250 \mu \mathrm{H}$, um capacitor de filme de polipropileno de $5 \mu \mathrm{F}$ e um resistor de amortecimento $R_{d}$ de $5 \Omega$. Com estes componentes resulta numa frequência de corte de $2,9 \mathrm{kHz}$ e amortecimento de $\xi=0,28$. O custo do filtro LC é aproximadamente um sexto do custo do banco de capacitores e também ocupa menos espaço como pode ser observado na Figura 17. Além disso, o capacitor de filme é mais estável e confiável do que os capacitores eletrolíticos, a longo prazo. Outra vantagem de substituir o banco de capacitores por um filtro LC é que o circuito de précarga do capacitor para proteger a fonte de tensão do inversor da corrente de pico quando o sistema é ligado

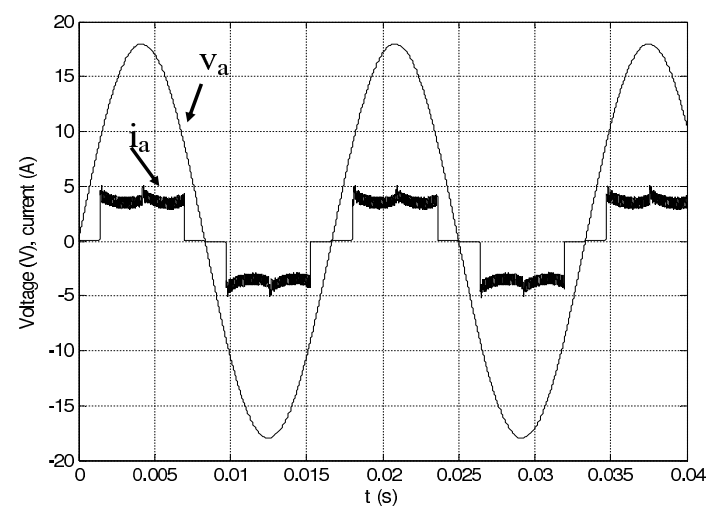

Figura 15: Tensão e corrente de entrada do inversor sem capacitores no elo de corrente contínua.

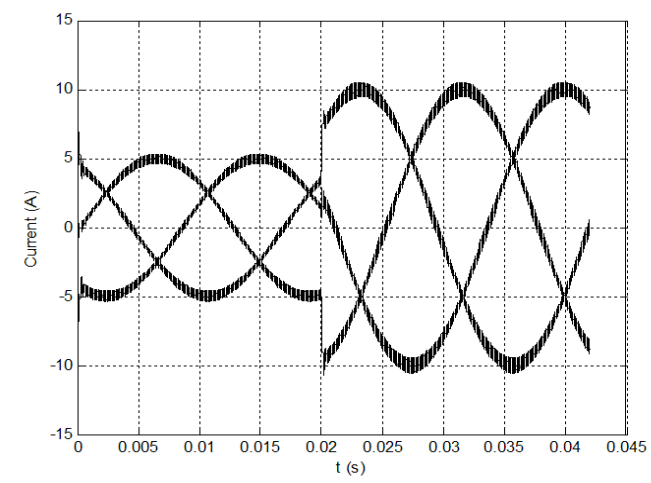

Figura 16: Resposta transitória do inversor sem capacitores no elo de corrente contínua com o controlador de corrente proposto.

ou para um evento de perda de linha é desnecessário.

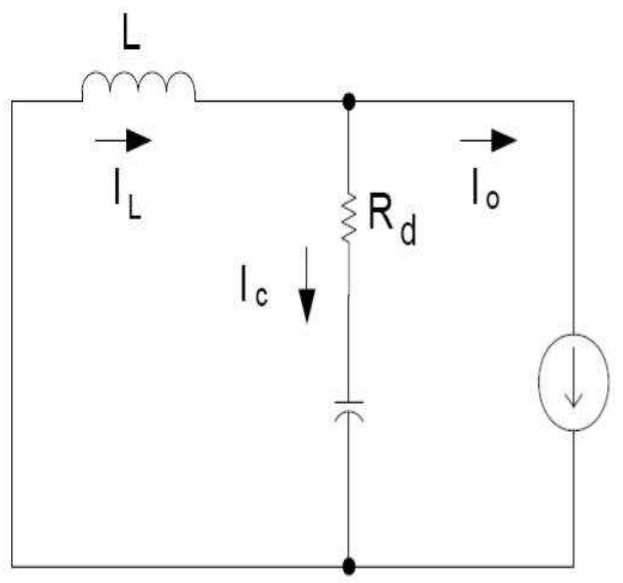

Figura 18: Circuito harmônico equivalente. 


\subsection{Circuito de Proteção}

O circuito de proteção de sobrecorrente é obtido a partir de dois circuitos comparadores que injetam um sinal de acionamento da proteção no módulo de potência IRAMS10UP60A (IRAMS10UP60A, n.d.). Quando ocorre um curto circuito todas as chaves são desabilitadas. Um problema surge quando a proteção de curtocircuito é ativada pois aparecerá uma sobretensão no elo de corrente contínua pois a carga normalmente é indutiva e não há um capacitor grande para absorvê-lo. Esta sobretensão pode destruir os semicondutores de potência. Para evitar esta sobretensão, um circuito clamp varistor é acrescentado ao elo de corrente contínua. Varistores podem responder a mudanças na tensão quase que instantaneamente. Dois parâmetros são importantes na seleção do varistor: tensão de grampeamento e a capacidade de absorver energia. No caso de uma carga $R L$, a energia armazenada $E$ é dada por (8).

$$
E=\frac{3}{4} L I^{2}
$$

Sendo que I é o valor eficaz da corrente de carga.

\subsection{Limitação do protótipo}

Para uma carga RL, os protótipos construídos foram suficientes para obtenção de alguns resultados experimentais. No entanto, a forma mais comum de carga para esses tipos de conversores é um motor trifásico de corrente alternada, que pode ser submetido a processos mecânicos ou elétricos de frenagem para a redução de velocidade. Em ambos os casos, o inversor fonte de tensão tem administrar a energia gerada pela energia cinética armazenada no motor e da carga. Se a inércia da carga for pequena ou o tempo de desaceleração não for tão pequeno, em uma fonte de tensão com um capacitor grande no elo de corrente contínua, é possível que o capacitor absorva a energia produzida sem um aumento significativo da tensão do elo de corrente contínua. Como não é o caso, é necessário um circuito de frenagem dinâmica ou um inversor fonte de tensão. O circuito de frenagem dinâmica é essencial no caso em que o inversor fonte de tensão funciona sem um capacitor eletrolítico grande. Outro aspecto importante a ser investigado e implementado no inversor sem capacitor no elo de corrente contínua é a preocupação com a interrupção momentânea de energia (Jouanne et al., 1999) que em algumas aplicações industriais é desejável se manter algum grau de controle do processo durante este tipo de interrupção.

\section{RESULTADOS EXPERIMENTAIS}

Os resultados experimentais apenas com inversor de frequência fonte de tensão são apresentados para a validação do controlador proposto. Os parâmetros de carga e frequência de comutação são os mesmos utilizados nas simulações.

Os resultados experimentais do inversor de frequência fonte de tensão com banco de capacitores eletrolíticos operando com modulação por vetores espaciais em malha aberta são apresentados nas Figura 19 e Figura 20. A Figura 19 mostra a tensão e a corrente de saída de um inversor fonte de tensão com o banco de capacitores e a Figura 20 mostra a corrente de entrada e seu respectivo espectro harmônico.

O controlador proposto para o inversor de frequência fonte de tensão sem o banco de capacitores do elo de corrente contínua foi implementado de acordo com o esquema apresentado na Figura 4 e com referência de corrente igual a 1,6 A. A tensão e a corrente de saída e o espectro harmônico da corrente de saída são mostrados na Figura 21.

A tensão e a corrente de entrada são mostradas na Figura 22 e o espectro harmônico da corrente de entrada é mostrado na 23. Observa-se a satisfatória resposta do controlador proposto pois a referência de corrente é atendida.

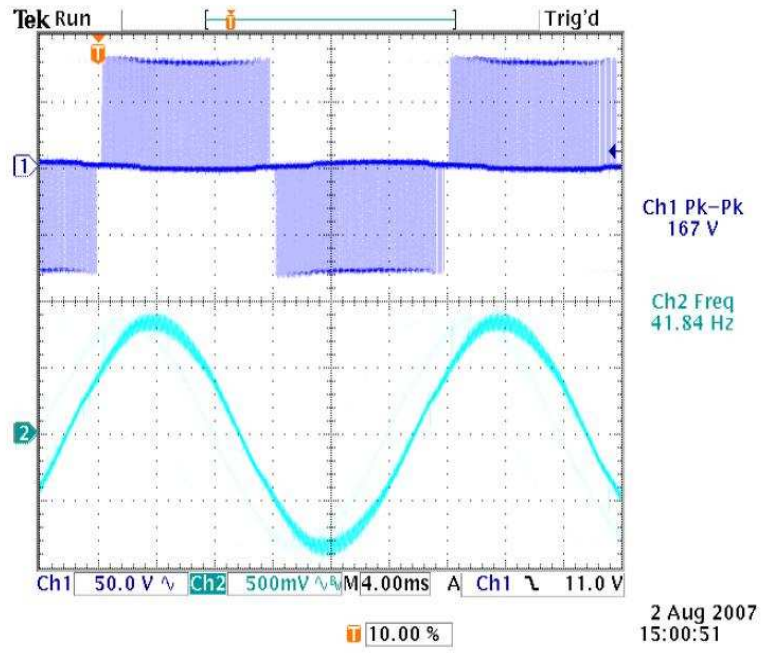

Figura 19: Tensão e corrente (2A/div) de saída do inversor de frequência com banco de capacitores. 

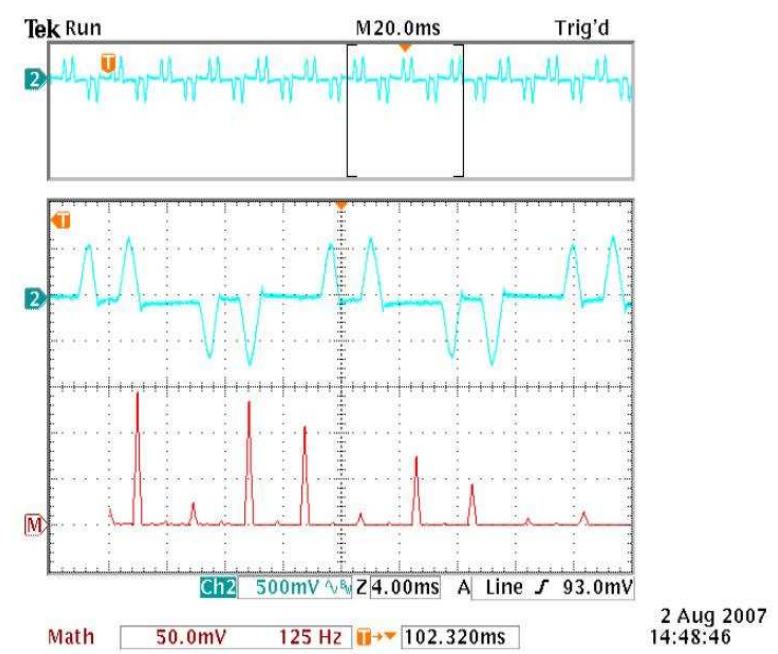

Figura 20: Corrente (2A/div) de entrada e seu espectro harmônico do inversor de frequência com banco de capacitores.

Comparando-se a Figura 19 com a Figura 21 pode-se observar a diferença entre a tensão de saída dos inversores provocada pela ausência do banco de capacitores no elo de corrente contínua embora a corrente de carga nos dois casos tenha formas semelhantes.

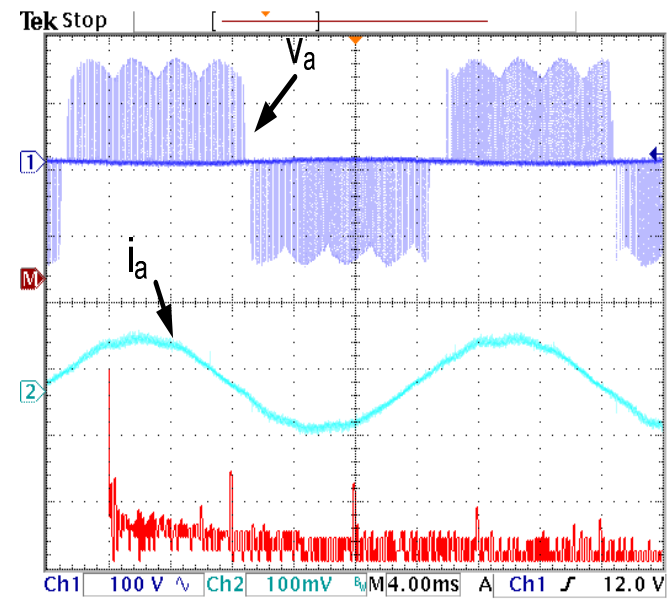

Math

$20.0 \mathrm{~dB} \quad 5.00 \mathrm{kHz} \mathrm{W} \rightarrow-7.04000 \mathrm{~ms}$ 27 Jul 2007

Figura 21: Tensão e corrente (2A/div) de saída do inversor de frequência sem banco de capacitores com o controlador proposto.

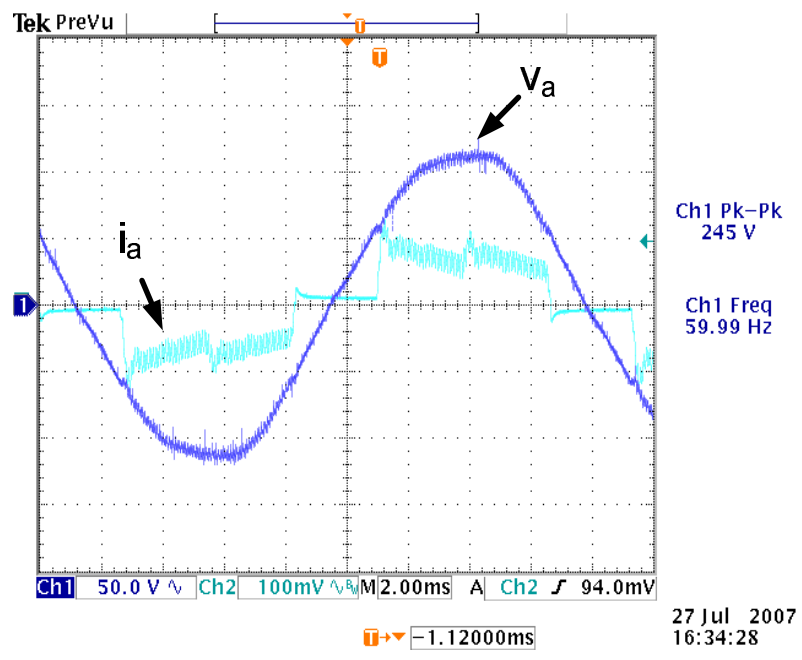

Figura 22: Tensão e corrente de entrada do inversor de frequência sem banco de capacitores com o controlador proposto.

Comparando-se a Figura 20 com a Figura 23 pode-se observar que houve uma drástica redução dos componentes harmônicos da corrente de entrada, quando o banco de capacitores eletrolíticos foi substituído pelo filtro LC.
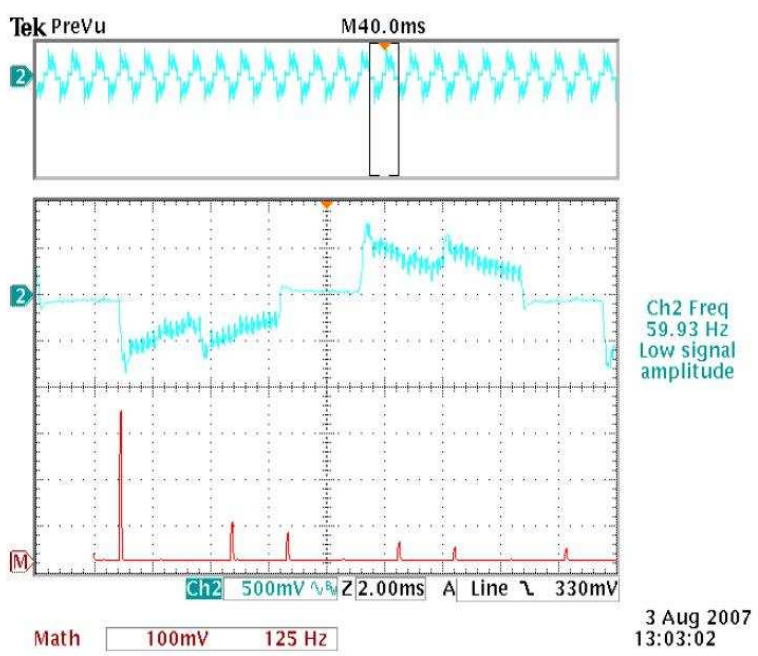

Figura 23: Corrente de entrada e seu espectro harmônico.

\section{CONCLUSÕES}

Este artigo apresentou uma proposta de controlador de corrente de carga para o CM e para o inversor de frequência fonte de tensão com a ausência do capacitor do elo de corrente contínua.

O controlador de corrente foi implementado com em- 
prego de um controlador PI usando modulação em largura de pulso por vetores espaciais o que possibilitou o uso de frequência de chaveamento constante. O controlador PI força que a amplitude do vetor espacial da corrente de saída seja constante através da manipulação do índice de modulação $m$. Assim, a referência de módulo do vetor corrente será atendida e as correntes de saída não terão componentes harmônicos de baixas frequências e também não serão desbalanceadas. Esta aplicação não tem alto custo computacional e a implementação de seu hardware não é complexa.

Com relação ao controlador de corrente quando aplicado ao CM, além das características citadas acima, pode-se afirmar que o controle também possibilita que as correntes da saída não se tornem desbalanceadas mesmo quando as tensões de entrada estão desbalanceadas.

O inversor fonte de tensão apresentou uma redução dos harmônicos na corrente de entrada quando o banco de capacitores eletrolíticos foi substituído pelo filtro LC. A tensão de saída do inversor tem uma forma semelhante à da tensão de saída do CM e isto é consequência da ausência do capacitor do elo de corrente contínua e da ação do controlador de corrente, embora a corrente de carga tenha uma forma similar à forma de corrente de carga do inversor fonte de tensão com banco de capacitores. O controle atua contra a flutuação indesejada da tensão do elo de corrente contínua, além de apresentar uma boa resposta transitória. A ausência do capacitor do elo de corrente contínua diminuiu o custo do inversor e a substituição do banco de capacitores por um filtro LC eliminou o circuito de pré-carga para proteger a fonte de tensão do inversor da corrente de pico durante a ligação do equipamento ou durante um evento de perda de linha.

A eficácia do controlador proposto foi verificada através de simulações digitais e resultados experimentais. Assim, o controlador de corrente pode ser aplicado aos inversores fonte de tensão sem capacitores no elo de corrente contínua e aos CM.

\section{AGRADECIMENTOS}

Os autores são gratos à CAPES - Coordenação de Aperfeiçoamento de Pessoal de Nível Superior e à Fapesp Fundo de Amparo à Pesquisa do Estado de São Paulo pelo suporte financeiro para esta pesquisa. Agradecem também, à Texas Instruments pela doação do DSP TMS320F2812.

\section{REFERÊNCIAS}

Accioly, A. G. H., Lima, V. N., Bradaschia, F., Neves, F. A. S., Cavalcanti, M. C. e Neto, A. S. (2007). Scalar pulse width modulation strategies for matrix converters, Eletrônica de Potência 12(1): 53 - 61 .

Basilio, C. e Matos, S. R. (2002). Design of pi and pid controllers with transient performance specification, IEEE Trans. On Education 45(4): 364-370.

Blaabjerg, F., Teodorescu, R., Liserre, M., e Timbus, A. V. (2006). Overview of control and grid synchronization for distributed power generation systems, IEEE Transactions on Industrial Electronics 53(5): 1398-1409.

Casadei, D., Serra, G. e Tani, A. (1998). Reduction of the input current harmonic content in matrix converters under input/output unbalance, IEEE Trans. on Industrial Electronics 45(3): 401-410.

Casadei, D., Serra, G., Tani, A. e Zarri, L. (2009). Optimal use of zero vectors for minimizing the output current distortion in matrix converters, IEEE Trans. on Industrial Electronics 56(2): 326 - 336.

Chen, X. e Kazerani, M. (2006). Space vector modulation control of na ac-dc-ac converter with a frontend diode rectifier and reduced dc-link capacitor, IEEE Trans. On Power Electronics 21(5): 14701478.

Choi, J. W. e Sul, S. K. (1997). New current control concept - minimum time current control in the threephse pwm converter, IEEE Trans. On Power Electronics 12(1): 124-131.

Enjeti, P. N. e Shireen, W. (1992). A new technique to reject dc-link voltage ripple for inverters operating on programmed pwm waveforms, IEEE Trans. On Power Electronics 7(1): 171-180.

Holtz, J. (1992). Scalar pulse width modulation strategies for matrix converters, IEEE Transactions on Industrial Electronics 39(5): 410-420.

Hubber, L. e Borojevic, D. (1995). Space vector modulated three-phase to three-phase matrix converter with input power factor correction, IEEE Trans. on Industry Appl. 31(6): 1234-1246.

IRAMS10UP60A (n.d.). data sheet, International Rectifier.

Jouanne, A. V., Enjeti, P. N. e Banerjee, B. (1999). Assessment of ride-through alternatives for adjustable-speed drives, IEEE Trans. On Ind. Applications 35(4): 908-916. 
Jussila, M. e Tuusa, H. (2007). Comparison of simple control strategies of space-vector modulated indirect matrix converter under distorted supply voltage, IEEE Trans. on Power Electronics 22(1): 139-148.

Kang, J. K., Hara, H., Watanab, E., Hava, A. M. e Kume, T. J. (2002). The matrix converter drive performance under abnormal input voltage, IEEE Trans. on Power Electronics 4(4): 234-240.

Kwak, S., Dabbousi, R. e Toliyat, H. (2002). An instantaneous compensation technique for supply disturbances in direct frequency converters, Proceedings of IECON 02 2: 956-960.

Liu, C., .Wu, Li, Y. e Wei, S. (2007). A novel threephase pwm rectifier/inverter without capacitor in dc-link, IEEE International Conference on Electrical Machines and Systems pp. 50 - 53.

Malesani, L., Rossetto, L., Tenti, P. e Tomasin, P. (1995). Ac/dc/ac pwm converter with reduced energy storage in the dc link, IEEE Trans. on Industry Appl. 31(2): 287-292.

Malesni, L. e Tomasin, P. (1993). Pwm current control techniques of voltage source converter- a survey, Proceedings of the IECON'93 2: 670-675.

Mutschler, P. e Marcks, M. (2002). A direct control method for matrix converters, IEEE Trans. on Industrial Electronics 49(2): 362-369.

Nielsen, P., Blaabjerg, F. e Pedersen, J. (1996). Space vector modulated matrix converter with minimized number of switchings and a feedforward compensation of input voltage unbalance, Proceedings of IEEE-PEDES '96 2: 833-839.

Nielsen, P., Casadei, D., Serra, G. e Tani, A. (1996). Evaluation of the input current quality by three different modulation strategies for svm controlled matrix converters with input voltage unbalance, Proceedings of IEEE-PEDES'96 (2): 794 -800.

Odaka, A., Sato, I., Ohgushi, H., Tamai, Y., Mine, H. e Itoh, J.-I. (2009). A pam control method for a matrix converter based on a virtual ac/dc/ac conversion method, Electrical Engineering in Japan 166(4): 88-96.

Rashid, M. (2004). Power electronics circuits, devices and applications, Pretince Hall.

Sato, I., ichi Itoh, J., Ohguchi, H., Odaka, A. e Mine, H. (2007). An improvement method of matrix converter drives under input voltage disturbances, IEEE Trans. on Power Electronics 22(1): 132-138.
Takeshita, T. e Shimada, H. (2008). Matrix converter control using direct ac/ac conversion approach to reduce output voltage harmonics, Electrical Engineering in Japan 162(2): 778 -787.

Vincenti, D. e Ziogas, P. D. (1992). An analysis and design of a force commutated three-phase pwm ac controller with input unbalance correction capability, Proceeding IEEE APEC'92 4(4): 487-493.

Wiechmann, E. P., Garcia, A. R., Salazar, L. D. e Rodriguez, J. R. (1997). High performance directfrequency converters controlled by predictive current loop, IEEE Trans. on Industrial Electronics 12(3): 547-557.

Zanchetta, P., Wheeler, P., Clare, J. e Bland, M. (2008). Control design of a three-phase matrix-converterbased ac-ac mobile utility power supply, IEEE Trans. on Industrial Electronics 55(1): 209 - 217.

Zhang, L., Watthanasaran, C. e Shepherd, W. (2001). Control of ac-ac matrix converter for unbalanced and/or distorted supply voltage, Proceedings of PESC'01 pp. 1108-1113. 